\title{
Hemorheological Effects of Valsartan in L-NAME Induced Hypertension in Rats
}

\author{
Ana Santos Silva-Herdade* and Carlota Saldanha
}

\author{
Instituto de Bioquímica, Unidade de Biologia Microvascular e Inflamação, Instituto de Medicina Molecular, Faculdade \\ de Medicina de Lisboa, Portugal
}

\begin{abstract}
Animal models are a useful tool, for example, in the study of hypertension, but its use requires robust knowledge and well characterized models in order to extrapolate the obtained results. Nitric oxide is an important regulator of vascular function and blood pressure. The chronic administration of nitric oxide synthase (NOS) inhibitors provides an animal experimental model of hypertension. Our study aims to investigate the hemorheological effects of (i) $N^{\omega}$-nitro-L-arginine methyl ester (L-NAME) added daily to Sprague-Dawley rats in drinking water and (ii) valsartan, an angiotensin II AT1-receptor antagonist, administration when the Sprague-Dawley rats acquired the hypertensive state. In three experimental groups (Control, Hypertensive and Valsartan) systolic blood pressure was measured and blood samples were collected for determination of erythrocyte membrane deformability and fluidity as well as blood and plasma viscosities.

L-NAME intake induces an increase in the systolic blood pressure indicating the development of systemic hypertension. Concerning the hemorheological parameters, the erythrocyte deformability is decreased in the hypertensive animal group and on contrary the erythrocyte membrane fluidity increased being both parameters values reverted after valsartan administration. Valsartan also decreases the plasma and blood viscosities values obtained in the animal group which have acquired systemic hypertension after L-NAME intake.

In conclusion, the angiotensin II AT1-receptor antagonist, valsartan, restores in hypertensive Sprague-Dawley rats LNAME- dependent their systolic blood pressure to the physiological values, as well as, normalized their hemorheological parameters values; due to the similarity effects to human essential hypertension we conclude that this can be a suitable animal model for hemorheological studies in the field of hypertension.
\end{abstract}

Keywords: Hypertension, hemorheology, animal model, nitric oxide synthase.

\section{INTRODUCTION}

Hypertension is a common disease among adult populations and is a significant contributor to ill health, resulting in an excess of both morbidity and mortality. Moreover, hypertension is directly implicated in various cardiovascular disease states, including stroke, ischemic disease and peripheral vascular disease [1]. Studies in the area of hypertension showed that, an increase in arterial blood pressure leads to a decrease in the nitric oxide for the circulation, which reflects the role of the arginin-nitric oxide in the pathophysiology of hypertension [2].

Hypertension is an important risk factor for cardiovascular diseases and its treatment is essential for the prevention of those illnesses. Hemorheological properties are known to be associated with hypertensive states, although it is still not known if hemorheological abnormalities are the cause or the result of systemic arterial hypertension [2]. There is a significant positive correlation between blood pressure and hematocrit value $[2,3]$ and it has been shown that whole blood viscosity, plasma viscosity and red blood

*Address correspondence to this author at the Instituto de Bioquímica, Unidade de Biologia Microvascular e Inflamação, Edíficio Egas Moniz, Faculdade de Medicina de Lisboa, 1649-028 Lisboa, Portugal;

Tel: +351217985136; Fax: +351217999477;

E-mail: anarmsilva@fm.ul.pt cell (RBC) aggregation tendency were increased, while erythrocyte deformability was decreased in hypertensive patients [2, 3]. On one hand, these hemorheological alterations may lead to an increased arterial blood pressure by altering the hemodynamic resistance $[4,5]$. On the other hand, erythrocyte redox properties and hemorheological parameters are well known to be sensitive to the disturbances in local and general homeostasis in the living organism [6].

There is evidence that in human blood circulation, erythrocyte acts either as a donor or a scavenger of nitric oxide (NO) mediated by $\mathrm{R}$ (oxygenated) or $\mathrm{T}$ (deoxygenated) states of haemoglobin in dependence of tissues oxygen partial pressure [7,8]. It has been also reported that NO modulated erythrocyte signal transduction mechanisms and deformability [9-13].

Animal models are important tools for the study of hypertension, but the use of laboratory animals implies a well characterized model in which it should be possible to generalize the findings and extrapolate them to other animal species or human [14]. This work aimed a hemorheological characterization and an erythrocyte nitric oxide efflux ability of an induced animal model of hypertension based on the chronic inhibition of NOS by the $N$-nitro-L-arginine methyl ester (L-NAME) and its response to the administration of an antagonist of AT1 receptors of angiontensin II, valsartan. 


\section{METHODS}

\subsection{Animals}

The animals used in this study received human care in accordance with the Directive of the European Community $n^{\circ} 86 / 609 / C E E$ that mentions the protection of animals used for economic ends and other scientific ends.

Sprague-Dawley male rats $(\mathrm{N}=30)$ (Rattus norvegicus, Sprague-Dawley, Harlan Iberica, Spain), with an average weight of $257 \pm 56 \mathrm{~g}$, were kept in an animal facility with a $12 \mathrm{~h}$ light/dark cycle and housed in cages in a temperature controlled room. All animals were kept on a diet standard rat food and water ad libitum. Three groups of animals were considered: a Control group $(\mathrm{N}=10)$, a Hypertensive group $(\mathrm{N}=10)$ and the Valsartan group $(\mathrm{N}=10)$. The Hypertensive group (HTA) was obtained in rats to which was given $600 \mathrm{mg} / \mathrm{L}$ of $N$-nitro-L-arginine methyl ester (L-NAME, Sigma, Fluka) in drinking water during 21 consecutive days, as in [15] and blood collections were performed at the end of this period. In the third group, valsartan group (HTA-VLT), rats were given $600 \mathrm{mg} / \mathrm{L}$ L-NAME in drinking water during 21 consecutive days, as in the previous group, and an intravenous injection of $2 \mathrm{mg} / \mathrm{Kg}$ of valsartan (Novartis, Germany), an antagonist of AT1-receptors of angiotensin II; in this group blood was collected 30 minutes after valsartan administration. Valsartan was administrated intravenous because it induces less stress and the dose administrated is comparable (dose/body weight) with the oral dose usually prescribed to humans

\subsection{Surgical Procedures}

For the surgical procedures and microcirculatory measurements, the rats were anesthetized intraperitoneally with $1,5 \mathrm{~g} / \mathrm{Kg}$ body weight urethane (Sigma-Aldrich) and intramuscular $50 \mathrm{mg} / \mathrm{Kg}$ body weight ketamine (Pfizer, Parke Davis) after 20 minutes. Body temperature was maintained between $35-37^{\circ} \mathrm{C}$ with auto-regulable heating platform. A tracheostomy was performed to maintain the animal in spontaneous breath during all the experiment. For drug administration, the right jugular vein was cannulated with polyethylene tubing and the left carotid artery was cannulated for measurement of mean arterial pressure and cardiac frequency. Blood was also collected in the left carotid. At the end of the experiments the animals were euthanized with over-dosage of anaesthesia.

\subsection{Systolic Blood Pressure}

Arterial pressure was measured with a catheter connected to a pressure transducer TRANSPAC $\AA$ (Abbot, Sligo, Ireland); being the cardiac frequency and the systolic and diastolic pressures measured and registered through hardware and software system PowerLab/400 (AD Instruments, Castle Hill, Australia).

\subsection{Measurement of NO by an Amperometric Method Error! Reference source not found.}

Erythrocyte suspensions were centrifuged and sodium chloride $0.9 \%$ at $\mathrm{pH} 7.0$ was added in order to reach a hematocrit of $0.05 \%$. The suspension was gently mixed by tube inversion. For amperometric NO quantification, we used the amino-IV sensor (Innovative Instruments Inc. FL, USA), as previously described [11]. Briefly, after stabilization of the NO sensor immersed in erythrocyte suspensions $(\mathrm{N}=5)$, the erythrocytes were stimulated with acetylcholine (ACh) $10 \mu \mathrm{M}$ and changes in the electric current registered, the change being proportional to the amount of NO mobilized by ACh-stimulated erythrocytes.

\subsection{Erythrocyte Deformability}

The erythrocyte deformability for different shear stress $(0.30,0.60,1.20,3.00,12.00,30.00$ and $60.00 \mathrm{~Pa})$ was determined by using the Rheodyn SSD shear stress diffractometer from Myrenne GMBH (Roentgen, Germany) and erythrocyte deformability is expressed as the elongation index (EI) in percentage. The Rheodyn SSD diffractometer determines RBC deformability by simulating the shear forces exerted by the blood flow and vascular walls on the erythrocytes [16, 17]. Erythrocytes are suspended in a viscous medium and placed between a rotating optical disk and a stationary disk. A well defined shear force is exerted upon the suspension which forces the erythrocytes to deform to ellipsoids and align with the fluid shear stresses. If a laser beam is allowed to pass through the erythrocyte suspension a diffraction pattern appears on the opposite end. That diffraction pattern will be circular with resting erythrocytes, but becomes elliptical when the erythrocytes are deformed by shear. The light intensity of the diffraction pattern are measured at two different points (A and B), equidistant from the center of the image. The erythrocyte elongation index (EEI), in percentage, is obtained according the following formula: $\mathrm{EEI}(\%)=\mathrm{A}-\mathrm{B} / \mathrm{A}+\mathrm{B} \times 100$.

\subsection{Erythrocyte Membrane Fluidity}

Erythrocyte membrane fluidity was assessed, as previously described [18]. using the lipophilic fluorescent probes DPH and TMA-DPH by fluorescence anisotropy measurements [19]. DPH fluorescence measurements were performed at an excitation wavelength $\left(\lambda_{\text {exc }}\right)$ of $352 \mathrm{~nm}$, an emission wavelength $\left(\lambda_{\mathrm{em}}\right)$ of $430 \mathrm{~nm}$ and both bandwidths of $5 \mathrm{~nm}$. TMA-DPH fluorescence measurements were carried out with $\lambda_{\text {exc }}=340 \mathrm{~nm}, \lambda_{\mathrm{em}}=425 \mathrm{~nm}$ and both bandwidths of 5 $\mathrm{nm}$. Results are expressed in fluorescence anisotropy values.

\subsection{Plasma and Blood Viscosity}

Blood samples treated with the anticoagulant $\mathrm{K}_{3}$ EDTA were centrifuged at $1200 \mathrm{rpm}$ for 1 minute. The resulting plasma was collected for the determination of plasma viscosity by the Harkness method [20]. Whole blood viscosity (WBV) was determined in a Brookfield digital viscometer model LVTDV II cp., using native blood aliquots submitted at low $\left(22.5 \mathrm{~s}^{-1}\right)$ and high $\left(225 \mathrm{~s}^{-1}\right)$ shear stress forces.

\subsection{Statistical Analysis}

Data are expressed as means \pm standard deviation. Oneway ANOVA tests were used to compare values between different groups. Statistical analysis was conducted using the GraphPadPrism version 5.0. Statistical significance was set at $\mathrm{p}<0.05$.

\section{RESULTS}

Systolic blood pressure was measured in the three experimental groups - Control, HTA and HTA-VLT in order to analyse the hypertensive state of all the experimental 

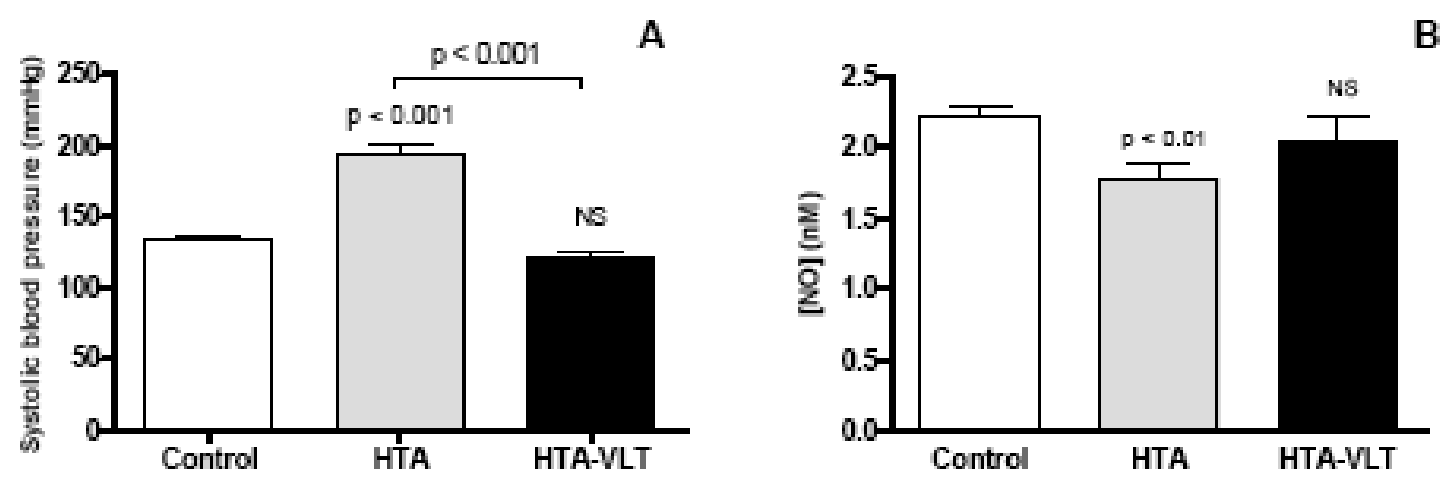

Fig. (1). (A) Mean \pm standard deviation of systolic blood pressure values in Control, HTA and HTA-VLT groups. (B) Mean values \pm standard deviation of NO concentrations in red blood cells in Control, HTA and HTA-VLT groups.

groups. Systolic blood pressure (Fig. 1) is significantly increased $(\mathrm{p}<0.001)$ relatively to the control in the HTA group, as already shown in [14] and significantly decreased $(\mathrm{p}<0.001)$ when compared with HTA group after administration of the antihypertensive (HTA-VLT group).

The NO concentrations in the red blood cells in the three experimental groups are represented in Fig. (1) showing, as expected, that NO concentration is significantly decreased $(\mathrm{p}<0.01)$ in the HTA group. The intravenous administration of valsartan increases the NO concentrations when compared with HTA group, to values similar to the Control group.

The, already known, association of hemorheological parameters with hypertension lead us, in this animal model of hypertension, to study some of those properties, in order to characterize this model. Erythrocyte deformability (Fig. 2) is, relatively to the normotensive, decreased in hypertensive rats (HTA), meaning that in this group the erythrocytes are less deformable, as already described by [14]. Analysing the values of EEI in the HTA-VLT group we observe that valsartan reverts the effect obtained in the hypertensive rats for higher values of shear stress, but maintained the deformability for lower shear stress levels in comparison with the HTA group.

The fluorescence anisotropy was determined in order to assess the erythrocyte membrane fluidity, high levels of fluorescence anisotropy represent low erythrocyte membrane fluidity. Therefore, hypertensive rats (HTA) have higher membrane fluidity when compared with the normotensive rats and after valsartan administration the erythrocyte membrane fluidity decreases to values similar with the control group (Fig. 3).

Both plasma and blood viscosities (Fig. 4) are significantly increased in the hypertensive rats group (HTA) and significantly decreased after valsartan administration. Althought not determined by us, L-NAME induced hypertension doesn't induce significant changes in hematocrit values [21], meaning that the changes in blood viscosity aren't a result of hematocrit changes.

\section{DISCUSSION}

Our results show that with this animal model of hypertension achieved by NOS inhibition, with L-NAME, leads to a hypertensive state, characterized by an increased systolic blood pressure, which is reverted by the antihypertensive - valsartan - administration.

Concerning erythrocyte NO efflux levels obtained in the induced - hypertensive animals there was a decrease in relation to the control group. There was NO efflux values reversion by administration of valsartan in that animal group after the installed hypertension leading to NO concentrations similar to normal levels. The decrease of erythrocyte NO efflux verified when hypertension was induced in the rats is not in accordance with our results obtained in blood samples of hypertensive humans [13]. The hypertension aetiology differences between human and L-NAME inducedhypertension animal model may be an explanation for the different compensatory mechanism of erythrocyte $\mathrm{NO}$ efflux. Valsartan is an angiotensin II type 1 (AT1) receptor blocker which doesn't reduce angiotensin II concentrations, in contrast with angiotensin converting enzyme inhibitors. Angiotensin II, by type 2 receptors, stimulates NO release by the endothelium [22-24], inducing vasodilation and decreasing the blood pressure. We may raise the hypothesis of a similar signal transduction mechanism induced by valsartan at erythrocyte level as we have demonstrated with

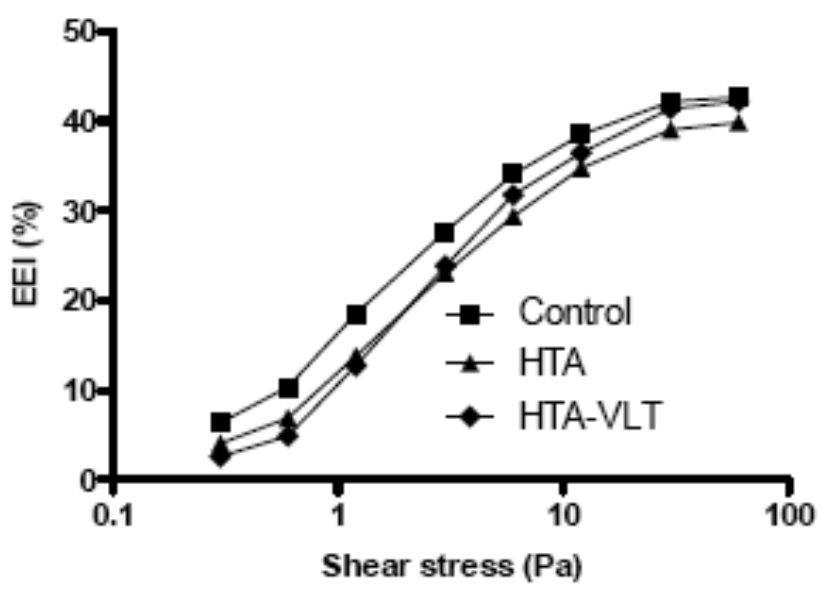

Fig. (2). Mean values of erythrocyte elongation index (EEI) in Control, HTA and HTA-VLT groups. 


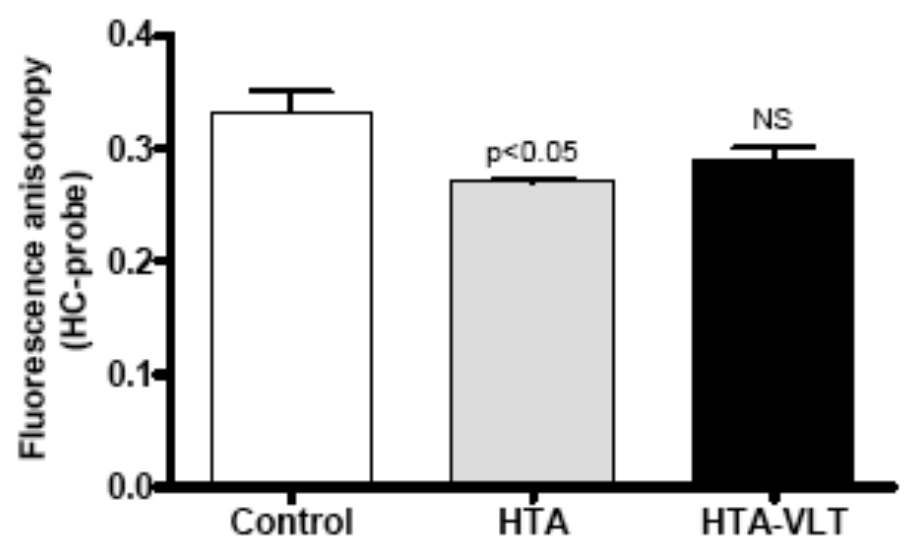

Fig. (3). Mean values \pm standard deviation of fluorescence anisotropy (HC probe) in Control, HTA and HTA-VLT groups.
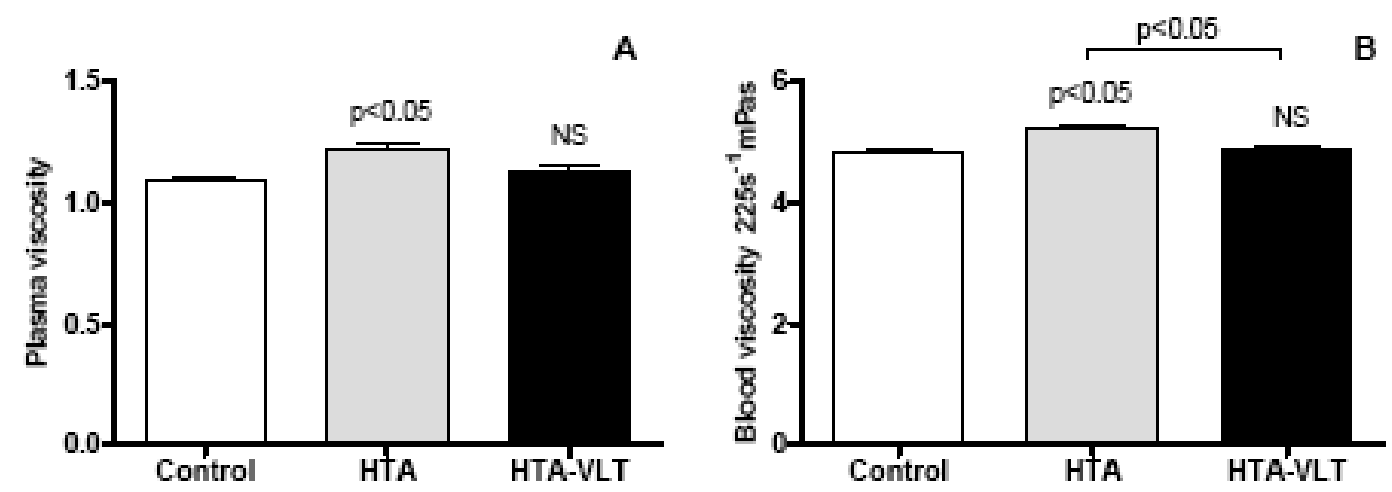

Fig. (4). Mean values \pm standard deviation for plasma (A) and blood viscosity (B) in Control, HTA and HTA-VLT experimental groups.

acetylcholine also known as a vasodilator mediated by $\mathrm{NO}$ at intact endothelium [11].

Hypertension is an important risk factor for cardiovascular diseases and that its treatment is essential for the prevention of those diseases. The study of hemorheological properties is very important in cardiovascular diseases and alterations in some hemorheological parameters, as blood and plasma viscosity, red blood cells deformability and aggregation, are associated with hypertensive conditions [3].

Relatively to the hemorheological properties of the NOS inhibition-induced hypertensive animal model there have similar profile to the hemorheological properties evaluated in humans with hypertension. Red blood cells deformability is lower after NOS inhibition which is reverted, for higher shear stress values, after the administration of valsartan. As a compensatory mechanism for the decreased deformability, in the hypertensive group the red blood cells membrane fluidity is increased. We verified that Valsartan in turn decreases the red blood cells membrane fluidity in hypertensive rats recovering the mice normal values. The mechanism underlying the valsartan induced-erythrocyte membrane fluidity changes may be associated with several biochemical process involving external domains of the erythrocyte membrane near the lipid-water [19]. Concerning plasma and blood viscosities, we found similar alterations as has been observed in human hypertensive patients [5], namely they are increased in the studied animal model of hypertension but both parameters values are decreased after valsartan administration.

NOS inhibition-induced hypertension animal model has a hemorheological profile similar to human essential hypertension [5] and valsartan ameliorates the hypertensive state restoring the hemorheological modifications obtained. These results show that the NOS inhibition-induced hypertension animal model is a suitable model for hemorheological studies in the field of hypertension, because the alterations observed in this model are similar to the ones observed in human essential hypertension and the reversibility of the model can easily be achieved by the administration of an antagonist of AT1-receptor of angiotensin II, like valsartan.

\section{ACKNOWLEDGEMENTS}

We thank Mrs. Teresa Freitas (Faculdade de Medicina Universidade de Lisboa) for the technical assistance in the hemorheological determinations.

\section{REFERENCES}

[1] Lip, G.Y.; Barnett, A.H.; Bradbury, A.; Cappuccio, F.P.; Gill, P.S.; Hughes, E.; Imray, C.; Jolly, K.; Patel, K. Ethnicity and cardiovascular disease prevention in the United Kingdom: a practical approach to management. J. Hum. Hypertens., 2007, 21, 183-211. 
[2] Kang, K.-T.; Sullivan, J.C.; Sasser, J.M.; Imig, J.D.; Pollock, J.S. Novel nitric oxide synthase-dependent mechanism of vasorelaxation in small arteries from hypertensive rats. Hypertension, 2007, 49, 893-901.

[3] Ross, R. Atherosclerosis - an inflammatory disease. N. Engl. J. Med., 1999, 340, 115-126.

[4] Chae, C.U.; Lee, R.T.; Rifai, N.; Ridker, P.M. Blood pressure and inflammation in apparently healthy men. Hypertension, 2001, 38, 399-403.

[5] Meiselman, H.J. Hemorheologic alterations in hypertension: chicken or egg? Clin. Hemorheol. Microcirc., 1999, 21, 195-200.

[6] Ajmani, R.S. Hypertension and hemorheology. Clin. Hemorheol. Microcirc., 1997, 17, 395-484.

[7] MacMahon, T.; Doctor, A. Extrapulmonary Effects of Inhaled Nitric Oxide. Proc Am. Thorac. Soc., 2006, 3, 153-160.

[8] Pawloski, J. R.; Hess, D. T.; Stamler, J. S. impaired vasodilation by red blood cells in sickle cell disease. PNAS 2005, 102, 2531-2536.

[9] Mallozzi, C.; Di Stasi, A.M.; Minetti, M. Peroxynitrite modulates tyrosine-dependent signal transduction pathway of human erythrocyte band 3. FASEB J., 1997, 11, 1281-1290

[10] Carvalho, F.A.; Mesquita, R.; Martins-Silva, J.; Saldanha, C. Acetylcholine and choline effects on erythrocyte nitrite and nitrate levels. J. Appl. Toxicol., 2004, 24, 419-427.

[11] Carvalho, F.A.; Martins e Silva, J.; Saldanha, C. Amperometric measurements of nitric oxide in erythrocytes. Biosens. Bioelectron., 2004, 20, 505-8.

[12] Mesquita, R.; Pires, I.; Saldanha, C.; Martins-Silva, J. Effects of acetylcholine and spermineNONOate on erythrocyte hemorheologic and oxygen carrying properties. Clin. Hemorheol. Microcirc., 2001, 25, 153-163.

[13] Carvalho, F.A.; Maria, A.V.; Braz Nogueira, J.M.; Guerra, J.; Martins-Silva, J.; Saldanha, C. The relation between the erythrocyte nitric oxide and hemorheological parameters. Clin. Hemorheol. Microcirc., 2006, 35, 341-347.
[14] van Zutphen, L.F.M.; Baumans, V.; Beynen, A.C. Principles of laboratory animal science. New York: Elsevier; 2005.

[15] Hacioglu, G.; Yalcin, O.; Bor-Kucukatay, M.; Ozkaya, G.; Baskurt, O.K. Red blood cell rheology properties in various rat hypertension models. Clin. Hemorheol. Microcirc., 2002, 26, 27-32.

[16] Starzyk, D.; Korbut, R.; Gryglewski, R.J.; Effects of nitric oxide and prostacyclin on deformability and aggregability of red blood cells of rats ex vivo and in vitro. J. Physiol. Pharmacol., 1999, 50, 629-637.

[17] Schmid-Schönbein, H.; Ruef, P.; Linderkamp, O. The shear stress diffractometer Rheodyn SSD for determination of erythrocyte deformability, I. Principle of operation and reproducibility, Clin. Hemorheol. Microcirc., 1996, 16, 48-50.

[18] Saldanha, C.; Santos, N.C.; Martins-Silva, J. Fluorescent probes DPH, TMA-DPH and C17-HC induced erythrocyte vesiculation. $J$. Membr. Biol., 2002, 190(1), 75-82.

[19] Lentz, B.R. Membrane 'fluidity' from fluorescence anisotropy measurements. Loew M Ed. Spectroscopic Membrane Probes, Volume I. Boca Raton (Florida): CRC Press; 1988.

[20] Harkness, J. A new instrument for measurement of plasma viscosity. Lancet 1963, 16, 280-281.

[21] Balaszczuk, A.M.; Tomat, A.; Bellucci, S.; Fellet, A.; Arrauz, C. Nitric oxide synthase blockade and body fluid volumes. Braz. J. Med. Biol. Res., 2002, 35, 131-134.

[22] Ruilope, L.M.; Rosei, E.A.; Bakris, G.L.; Mancia, G.; Poulter, N.R.; Taddei, S.; Unger, T.; Volpe, M.; Waeber, B.; Zannad, F. Angiotensin receptor blockers: therapeutic targets and cardiovascular protection. Blood Pressure, 2005, 14, 196-209.

[23] London, M. The role of blood rheology in regulating blood pressure. Clin. Hemorheol. Microcirc., 1997, 17, 93-106.

[24] Mchedlishvili, G.; Tsinamdzvrishvili, B.; Beritashvili, V.; Gobejishvili, L.; Ilencko, V. New evidence for involvement of blood rheological disorders in rise of peripheral resistance in essential hypertension. Clin. Hemorheol. Microcirc., 1997, 17, 3139.

This is an open access article licensed under the terms of the Creative Commons Attribution Non-Commercial License (http://creativecommons.org/licenses/by-nc/3.0/) which permits unrestricted, non-commercial use, distribution and reproduction in any medium, provided the work is properly cited. 